\title{
Interrelation between heart failure with preserved ejection fraction and renal impairment
}

\author{
Jennifer Ruth Joslin ${ }^{1,2}$, Eirini Lioudaki ${ }^{1, *}$, Emmanuel Androulakis ${ }^{3}$ \\ ${ }^{1}$ King's Kidney Care, King's College Hospital, SE5 9RS London, UK \\ ${ }^{2}$ Centre for Nephrology, Urology and Transplantation, King's College London, SE5 9NU London, UK \\ ${ }^{3}$ Heart Imaging Centre, Royal Brompton and Harefield Hospitals, SW3 6NP London, UK \\ *Correspondence: eirini.lioudaki1@nhs.net (Eirini Lioudaki) \\ Academic Editor: Giuseppe Coppolino \\ Submitted: 15 November 2021 Revised: 5 January 2022 Accepted: 19 January 2022 Published: 18 February 2022
}

\begin{abstract}
Heart failure with preserved ejection fraction (HFpEF) and chronic kidney disease (CKD) are global diseases of increasing prevalence and are frequent co-diagnoses. The two conditions share common risk factors and CKD contributes to HFpEF development by a variety of mechanisms including systemic inflammation and myocardial fibrosis. HFpEF patients with CKD are generally older and have more advanced disease. CKD is a poor prognostic indicator in HFpEF, while the impact of HFpEF on CKD prognosis is not sufficiently investigated. Acute kidney injury (AKI) is common during admission with acute decompensated HFpEF, but short and long-term outcomes are not clear. Pharmacological treatment options for HFpEF are currently minimal, and even more so limited in the presence of CKD with hyperkalaemia being one of the main concerns encountered in clinical practice. Recent data on the role of sodium-glucose cotransporter 2 (SGLT2) inhibitors in the management of HFpEF are encouraging, especially in light of the abundance of evidence supporting improved renal outcomes. Herein, we review the pathophysiological links between HFpEF and CKD, the clinical picture of dual diagnosis, as well as concerns with regards to renal impairment in the context of HFpEF management.
\end{abstract}

Keywords: heart failure with preserved ejection fraction; chronic kidney disease; end-stage renal disease; acute kidney injury

\section{Introduction}

Heart failure with preserved ejection fraction (HFpEF) is increasingly prevalent across the world and comprises around half of all patients with clinical heart failure [1]. Diagnosis of HFpEF is considered in patients who are breathless, do not have reduced left ventricular ejection fraction (LVEF) (commonly taken as $\geq 50 \%$, but cut-off varies), and in whom other cardiac and non-cardiac causes for symptoms have been excluded, and consensus diagnosis recommendations have now been developed [2]. Chronic kidney disease (CKD) encompasses structural kidney abnormalities or reduced function over at least three months, and is also a growing global burden with a global prevalence of 9.1\% [3]. Both HFpEF and CKD are increasingly common at older ages, share a number of risk factors for development, and dual diagnosis is common and set to grow. In this paper we will review the pathophysiological links between HFpEF and CKD, prevalence and prognosis of dual diagnosis, evidence for pharmacological treatment options, and practical considerations for management of the co-existing conditions, and briefly review acute kidney injury (AKI) in patients with HFpEF.

\section{Pathophysiological processes linking HFPEF and CKD}

While common risk factors for HFpEF and CKD are clearly identified, including diabetes, hypertension and obesity, a number of pathophysiological processes contribute to the interplay between cardiac and renal dysfunction (Fig. 1), which are not completely understood.

Myocardial fibrosis is the key pathology seen in HF$\mathrm{pEF}$ [4], with resulting cardiac structural and functional abnormalities which can potentially affect kidney function. Right ventricular (RV) dysfunction has been implicated in the development of renal impairment in patients with heart failure with a reduced ejection fraction (HFrEF) for some time [5]. In HFpEF worse RV indices are seen in association with $\mathrm{CKD}$, often with poorer prognoses [6-8]. Left ventricular (LV) hypertrophy without dilatation is a further cardinal feature of HFpEF, and increased LV mass predicts progression of renal disease in community cohorts at high risk for heart failure $[9,10]$. Left atrial enlargement and diastolic dysfunction are common in HFpEF [11-13], and impaired left atrial function predicts renal function decline in patients seen in a tertiary cardiology clinic with dyspnoea [14] and left atrial strain predicts progression of renal disease in patients with CKD [15], although causality is uncertain.

Pulmonary hypertension is seen in the majority of patients with HFpEF [13], largely thanks to metabolic insult in addition to pressure-related injury [16]. Haemodynamic markers of pulmonary hypertension (elevated transpulmonary gradient, pulmonary vascular resistance, and diastolic pulmonary gradient) were associated with increased 


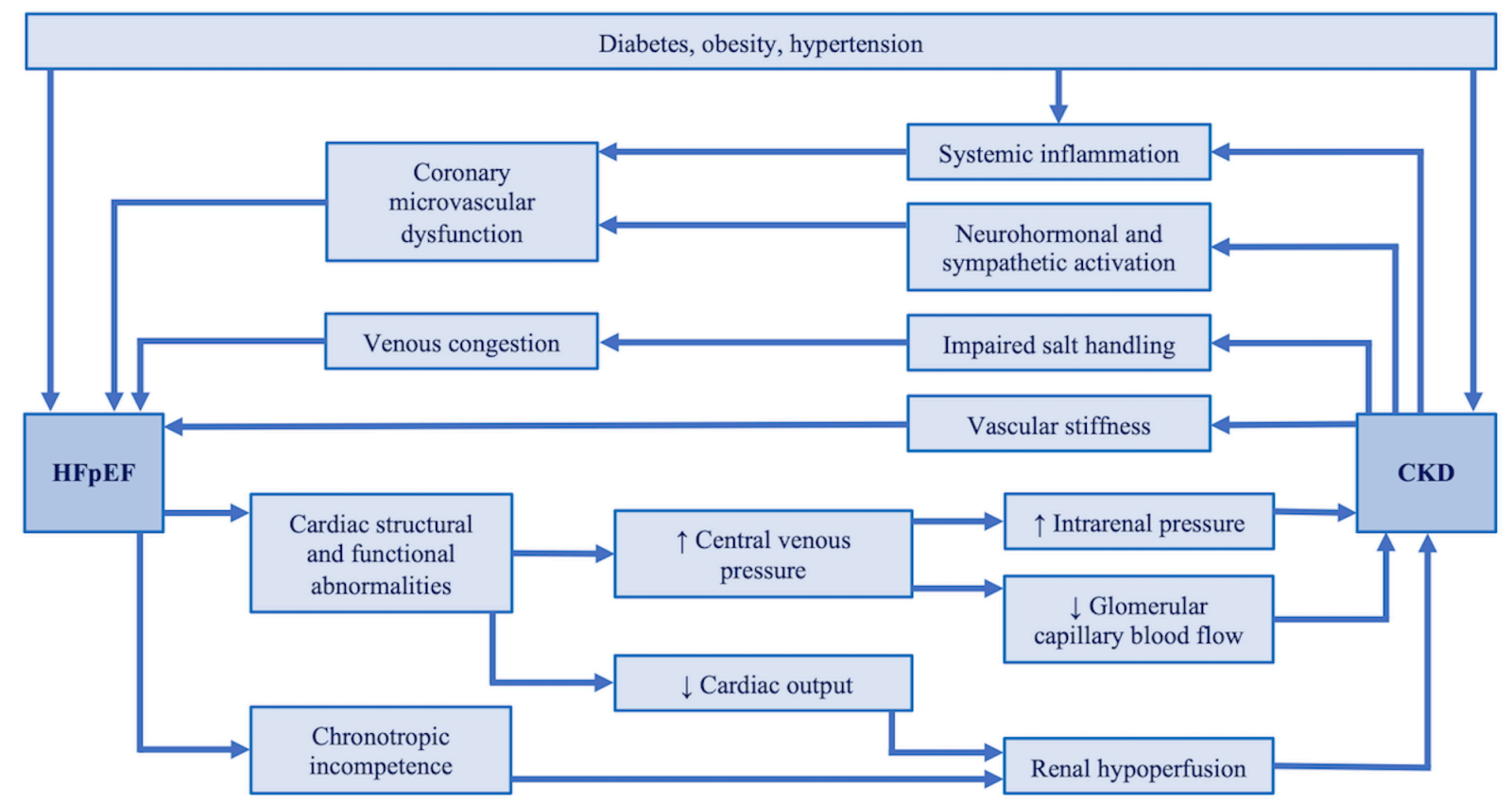

Fig. 1. Summary of pathophysiological processes linking heart failure with preserved ejection fraction (HFpEF) and chronic kidney disease (CKD). HFpEF and CKD share common risk factors including diabetes, hypertension and obesity, but also a number of pathophysiological processes contribute to the interplay between cardiac and renal dysfunction.

mortality and increased cardiac hospitalisations in patients with HFpEF from a large cohort undergoing right-heart catheterisation [17]. Pulmonary hypertension is recognised as increasing the risk of poorer outcomes after kidney transplantation $[9,10,18,19]$.

Furthermore, increased central venous pressure in HF$\mathrm{pEF}$ results in reduced glomerular capillary blood flow and raised interstitial and tubular pressures within the kidney [20]. On top of that, patients with HFpEF commonly have chronotropic incompetence, which is independently associated with reduced glomerular filtration rate (GFR) [21], likely due to renal hypoperfusion. Novel haemodynamic parameters may offer insights into further pathophysiologic mechanisms [22].

In the opposite direction, renal impairment is an independent risk factor for development of HFpEF [23]. Indeed, in a porcine model CKD via 5/6 nephrectomy induced development of HFpEF [24], while human data suggest that advanced CKD is associated with a variety of cardiac structural abnormalities [25]. Patients with CKD have impaired sodium handling and fluid overload, directly contributing to venous congestion, which is more pronounced in the advanced stages of the disease. Up to date understanding makes central the importance of systemic inflammation in HFpEF pathophysiology, leading to coronary microvascular endothelial inflammation and dysfunction, and ultimately to stiff cardiomyocytes and diastolic LV stiffness via reduced nitric oxide signalling [26]. Traditional HFpEF co-morbidities such as diabetes and hypertension contribute to the inflammation, and CKD itself is well-recognised as a pro-inflammatory state [27]. Obesity is over-represented in older patients with HFpEF and adipose tissue contributes to a number of pathophysiological pathways increasing inflammation and oxidative stress, potentially leading to both cardiac and renal dysfunction [28]. Further, there is some evidence that increased fibroblast growth factor 23 levels in CKD induce LV hypertrophy [29], while enhanced sympathetic activation in CKD can contribute to heart failure [30], with increased neurohormonal activation noted in both CKD and pulmonary hypertension being associated with vascular remodelling and worse outcomes [31].

It is also worth considering that in a minority of patients HFpEF may be caused by specific diseases, some of which, such as amyloidosis, may affect the kidneys concurrently.

\section{Definition of HFpEF}

The European Society of Cardiology 2019 consensus statement on the diagnostic criteria for HFpEF includes the definition of a "preserved" LVEF as $\geq 50 \%$ [2]. HFpEF was historically described as "diastolic heart failure", and the threshold for EF has not always been consistent. It is necessary when considering HFpEF studies and treatment trials to acknowledge these inconsistencies and highlight the HFpEF inclusion criteria and relevant EF-based sub-group analysis. We include a table of the most important trials discussed below, with the key details for each (Table. 1). 


\section{Prevalence and clinical picture}

CKD is commonly seen in HFpEF, although estimated prevalence varies. Most studies report CKD as estimated GFR (eGFR) $<60 \mathrm{~mL} / \mathrm{min} / 1.73 \mathrm{~m}^{2}$, corresponding to stage 3 CKD onward [32]. Recent studies have reported rates of CKD in HFpEF as $60 \%$ in a Belgian cohort [33], 50\% in a pan-Asia cohort [34] (both including LVEF $\geq 50 \%$ ), and $48 \%$ in the worldwide PARAGON-HF study [35] (LVEF $\geq 45 \%$ ). Albuminuria is also common in heart failure even without reduction in eGFR [36], making it likely that CKD is generally under-reported.

Multiple studies have shown that HFpEF patients with CKD have higher rates of traditional cardiovascular risk factors such as hypertension and diabetes than those without $[6,7,33,37]$, and the clinical picture of patients with HFpEF and co-existing CKD is generally that of more advanced disease.

HFpEF encompasses a broad range of clinical characteristics, possibly resulting from the multiple pathophysiological mechanisms, making understanding and prediction of renal involvement somewhat challenging. Phenomapping has allowed for distinct clusters of HFpEF patients to be classified. The first published study found that the cluster more likely to have CKD are older and have most severe electrical and myocardial remodelling, pulmonary hypertension, and RV dysfunction [8].

The cohort with CKD (eGFR $20-60 \mathrm{~mL} / \mathrm{min} / 1.73 \mathrm{~m}^{2}$ ) in the RELAX study (inclusion LEVF $\geq 50 \%$ ) had more advanced symptoms, more impaired indices of cardiac function and structure, and higher levels of biomarkers of neurohormonal activation, collagen metabolism/fibrosis and oxidative stress, when compared to those with normal kidney function. They also had lower exercise capacity, which was largely accounted for by lower haemoglobin levels [7].

A prospective study of $299 \mathrm{HFpEF}$ patients (with LVEF $>50 \%$ ) in the USA used conventional 2-D and speckle-tracking echocardiography to characterise cardiac structural and functional indices in HFpEF patients with and without CKD. As seen in other cohorts those with CKD (taken as $<60 \mathrm{~mL} / \mathrm{min} / 1.73 \mathrm{~m}^{2}$ ) were older, had a higher beta natriuretic peptide (BNP), were more likely to have diabetes and hypertension, and had a worse New York Heart Association (NYHA) functional class. Parameters of LV longitudinal strain, RV free wall strain and left atrial (LA) strain were associated with CKD, even after multivariate adjustment [6].

$\mathrm{HFpEF}$ is also common in patients with end-stage renal disease (ESRD) managed with maintenance haemodialysis (HD) or peritoneal dialysis (PD). Antlanger et al. [38] reviewed a cohort of 105 ESRD patients undergoing regular HD in Vienna, Austria, and found that $57 \%$ fulfilled the definition criteria of HFpEF (including LVEF $\geq 45 \%$ ), compared to 13 with HFrEF (LVEF <45\%). Predictors of HFpEF in this cohort included higher age, female sex, higher systolic blood pressure, and dialysis vintage, as we might expect, but interestingly not diabetes. Fluid overload (assessed by pre-dialysis bioimpedance spectroscopy) was not associated with heart failure, and nor were dialysisrelated factors including access via arteriovenous fistula, blood flow, and ultrafiltration volume. Of a further cohort of 1034 regular HD patients in Romania, 266 (26\%) had HFpEF (LVEF $\geq 50 \%$ ), compared to 118 with heart failure with mid-range ejection fraction (HFmrEF, LVEF 40-49\%) and 38 (4\%) with HFrEF (LVEF <40\%), although characteristics of the specific groups are not given [39]. Even without overt heart failure, structural heart abnormalities are common in ESRD. In a study of 654 patients starting HD, 567 (87\%) had echocardiographic evidence of structural heart disease, with diastolic dysfunction in 350 (78\% of the 448 in which diastolic function was reviewed) [25].

In a Chinese cohort of 'advanced CKD' patients - in practice a mix of CKD stage 4 and ESRD, including those on $\mathrm{HD}$ and $\mathrm{PD}$ - the HFpEF group (inclusion LVEF $\geq 50 \%$ ) were more likely than the non-HFpEF group to have diabetes and also had a higher systolic blood pressure [40]. Finally, among 220 patients undergoing regular PD in Hong Kong, 47 (21\%) had HFpEF (inclusion LVEF $\geq 50 \%$ ) and $39(18 \%)$ had HFrEF [41]. In this cohort higher age, presence of diabetes, background of coronary artery disease, and/or other atherosclerotic vascular disease, higher BNP and higher troponin T were all associated with HFrEF, with the HFpEF group generally intermediate between HFrEF and no heart failure. There were no differences observed in PD parameters.

Overall, patients with concomitant HFpEF and CKD appear to be characterised by a more severe disease burden as a result of both the presence of shared for the two conditions risk factors as well as the added impact of the two conditions.

\section{Prognosis}

It has been recognised for some time that prognosis is worse for HFpEF patients with concomitant renal dysfunction. Studies of patients admitted with acute decompensation of HFpEF report rates of baseline CKD far higher than those seen in the general HFpEF population, of up to $82 \%$ [42].

In a cohort of 397 patients identified post-discharge following hospital admission with symptomatic HFpEF, the pheno-group with higher rates of CKD had increased mortality and hospitalisation rate than those without [8]. The diagnostic cut-off for LVEF was $\geq 50 \%$, the mean reported LVEF was $61 \%$, without significant differences between pheno-groups. In a Belgian cohort of 183 patients $\mathrm{HFpEF}$ (inclusion $\mathrm{LVEF} \geq 50 \%$ ) and multivariate analysis showed that lower eGFR independently predicted the composite endpoint of all-cause mortality or hospitalisation for heart failure, and mortality alone [33]; this cohort was notably elderly (mean age $78 \pm 8$ years) and excluded those with NYHA functional class I disease. 
The Angiotensin Receptor Neprilysin Inhibition in Heart Failure With Preserved Ejection Fraction (PARAGON-HF) trial compared treatment with sacubitril/valsartan versus valsartan in 4796 patients with HFpEF over a median follow-up period of 34 months. Participants were all aged $\geq 50$ years and had NYHA functional class II-IV symptoms. Analysis of those enrolled showed a higher all-cause mortality risk for patients with eGFR $<60 \mathrm{~mL} / \mathrm{min} / 1.73 \mathrm{~m}^{2}$ compared to with eGFR $\geq 60 \mathrm{~mL} / \mathrm{min} / 1.73 \mathrm{~m}^{2}[43]$; it is important to note that patients with eGFR $<30 \mathrm{~mL} / \mathrm{min} / 1.73 \mathrm{~m}^{2}$ were excluded from the trial. PARAGON-HF also defined HFpEF as including participants with LVEF $\geq 45 \%$, although mean LVEF of enrolled participants was $57.6 \%$, and based on the presented standard deviation in each arm only a very small $\%$ of participants would have had LVEF less than the more usual $50 \%$ [35].

In line with PARAGON-HF findings, the analysis of the 1767 patients in the Americas group in the Treatment of Preserved Cardiac Function Heart Failure with an Aldosterone Antagonist Trial (TOPCAT) study highlighted an independent inverse relationship between eGFR and many clinical outcomes, including all-cause mortality and heart failure hospitalisations [37]. Again, patients with an eGFR $<30 \mathrm{~mL} / \mathrm{min} / 1.73 \mathrm{~m}^{2}$ were excluded, and HFpEF threshold included patients with LVEF $\geq 45 \%$. A recent secondary analysis of the TOPCAT cohort also observed that longitudinal variability of kidney function laboratory parameters (urea and creatinine) was independently associated with mortality and the composite endpoint of cardiovascular death, heart failure hospitalisation and aborted cardiac arrest [44]. Chirinos et al. [45] analysed samples from TOPCAT to consider potential prognostic biomarkers. For consideration of kidney function or injury, the panel include Neutrophil gelatinase-associated lipocalin (NGAL), Cystatin-C, and Kim-1, but none of these were predictive of the composite endpoint.

Proteinuria or albuminuria are signs of abnormal kidney function and can often be present before GFR drops. As such, a proportion of patients with CKD may be overlooked if the only criteria used to identify them is eGFR $\leq 60 \mathrm{~mL} / \mathrm{min} / 1.73 \mathrm{~m}^{2}$. Accordingly, some studies have specifically assessed their impact. Albuminuria without reduced GFR was associated with poorer outcomes and cardiac remodelling in a US cohort of 144 patients with $\mathrm{HFpEF}$ including LVEF $\geq 50 \%$ [46]. Further, the presence of dipstick proteinuria and albuminuria appear to be independent prognostic markers in HFpEF [47,48].

Information regarding prognosis in patients with concomitant ESRD and HFpEF is limited. In one small study of patients on regular HD, the presence of HFrEF was strongly associated with a higher risk of cardiac death and/or hospitalisation for cardiovascular reason adjusted hazard ratio (HR) 3.24; 95\% CI 1.08-9.75; $p=0.037$ ) but for HFpEF (including $\mathrm{LVEF} \geq 45 \%$ ) this association did not reach sta- tistical significance (adjusted HR 4.31, 95\% CI 0.46-40.03; $p=0.199$ ) [38]. A recent study of 1034 patients on regular HD in Romania found comparable survival (all-cause mortality) to four years between the groups with HFpEF and no heart failure, but reduced survival for patients with LVEF $<50 \%$ [39]. In PD, analysis of outcomes over four years showed that HFpEF (including LVEF $\geq 50 \%$ ) was associated with increased cardiac mortality, all-cause mortality, heart-failure events and cardiovascular events compared to those without heart failure, but for all outcomes the risk was significantly greater for those with HFrEF [41].

Overall, renal impairment appears to be associated with a worse prognosis in HFpEF patients. There is not specific data regarding the prognostic implications of $\mathrm{HFpEF}$ on CKD progression or mortality; nevertheless, a number of echocardiographic features typically seen in HFpEF have been associated with poorer renal outcomes and/or mortality in CKD [49].

\section{Management of patients with HFpEF and CKD, and practical considerations}

\subsection{General considerations}

Diagnosis of co-existing HFpEF and CKD can proceed along usual pathways, although in a community setting without access to echocardiography, then N-terminal-pro brain natriuretic peptide (NT-proBNP) is commonly used in cases of suspected heart failure, and a higher threshold is needed for diagnosis in patients with CKD [50].

Clinicians caring for patients in the advanced stages of CKD will be mindful that typical symptoms and signs such as shortness of breath, fatigue and peripheral oedema commonly occur in either condition, or distinguishing the underlying cause with dual pathology can be challenging. They should also be aware that the creation and use of upper limb arteriovenous fistulae and grafts (for dialysis in end stage renal disease) is associated with the development of right ventricular remodelling and dysfunction [51], potentially contributing to HFpEF.

There are similarities in the management of patients with HFpEF and CKD, including iron replacement in deficiency, optimal treatment of associated co-morbidities (particularly weight management, and control of diabetes and hypertension), and diuretics to treat fluid overload. Loop diuretics are the treatment of choice for troublesome peripheral oedema in both conditions, but the threshold dose is typically higher in CKD than in patients with normal renal function. Diuretic use in CKD may be associated with greater decline in renal function, even after other variables including hydration status and baseline eGFR are taken into account [52], and a careful balance is often needed.

Co-existing major coronary artery disease and atrial fibrillation should be managed, but specific treatment options for HFpEF are limited, especially in comparison to HFrEF. Treatments that are effective in HFrEF such as renin-angiotensin-aldosterone system (RAAS) inhibitors 
Table 1. LVEF criteria and LVEF sub-group analysis used in major HFpEF trials.

\begin{tabular}{|c|c|c|c|}
\hline Trial (intervention) & $\begin{array}{c}\text { HFpEF LVEF } \\
\text { criteria }\end{array}$ & $\begin{array}{c}\text { Mean LVEF of HFpEF } \\
\text { participants }\end{array}$ & Relevant sub-group analysis \\
\hline I-PRESERVE (irbesartan) & $\geq 45 \%$ & $59.50 \%$ & - \\
\hline $\begin{array}{l}\text { CHARM-Preserved (can- } \\
\text { desartan) }\end{array}$ & $>40 \%$ & $\begin{array}{l}\text { Not specified, but } \\
35.5 \% \text { of participants } \\
\text { had LVEF } 41-49 \%\end{array}$ & $\begin{array}{l}\text { Specific consideration of group with LVEF } 40-49 \% \text { showed benefit } \\
\text { of treatment with regard to primary outcome: HR } 0.76(95 \% \mathrm{CI} \\
0.61-0.96 ; p=0.02)\end{array}$ \\
\hline TOPCAT (spironolactone) & $\geq 45 \%$ & $57.10 \%$ & $\begin{array}{l}\text { Interaction between continuous LVEF and treatment for primary } \\
\text { outcome: } p=0.046 \text {; Interaction between continuous LVEF and } \\
\text { treatment for HF hospitalisations: } p=0.039\end{array}$ \\
\hline $\begin{array}{l}\text { PARAGON-HF } \\
\text { (sacubitril-valsartan) }\end{array}$ & $\geq 45 \%$ & $57.60 \%$ & $\begin{array}{l}\text { HR for events in sacubitril-valsartan arm in group with LVEF } \\
\leq \text { median, } 57 \%) \text { : } 0.78 \text { ( } 95 \% \text { CI } 0.64-0.95) \\
\text { HR in group with LVEF > median, } 57 \%: 1.00(95 \% \text { CI } 0.81-1.23)\end{array}$ \\
\hline RELAX (sildenafil) & $\geq 50 \%$ & $\begin{array}{l}61.3 \% \text { (calculated from } \\
\text { presented data) }\end{array}$ & - \\
\hline $\begin{array}{l}\text { EMPEROR-Preserved } \\
\text { (empagliflozin) }\end{array}$ & $>40 \%$ & $54.30 \%$ & $\begin{array}{l}\text { Treatment benefit with respect to primary composite in groups with } \\
\text { lower LVEF: } \\
\text { LVEF }<50 \% \text { HR } 0.71(95 \% \text { CI } 0.57-0.88) \\
\text { LVEF } \geq 50 \text { to }<60 \% \text { HR } 0.88(95 \% \text { CI } 0.64-0.99) \\
\text { LVE } \geq 60 \% \text { HR } 0.87 \text { (95\% CI } 0.69-1.10)\end{array}$ \\
\hline
\end{tabular}

and vasodilators have not shown the same benefit, e.g., in the HFpEF I-PRESERVE and CHARM-Preserved trials [53-56], likely reflecting the differing underlying pathophysiologies. A re-analysis of CHARM participants with LVEF 40-49\% suggested outcome benefits for candesartan in this range, but not when LVEF $\geq 50 \%$ [57]. HFpEF RAAS inhibition and vasodilation can drop preload and thus stroke volume to a degree that causes renal hypoperfusion. Other strategies are under consideration, with varying effects on renal function (Table. 2). It is likely that studies of treatments for HFpEF have been hampered by the heterogeneity of clinical characteristics.

\subsection{Mineralocorticoid receptor antagonists (MRAs)}

MRAs improve prognosis in HFrEF. Initial results of the (Treatment of Preserved Cardiac Function Heart Failure With an Aldosterone Antagonist) TOPCAT study of 3445 patients with symptomatic HFpEF (LVEF $\geq 45 \%$; mean $57.1 \%$ ) did not show outcome benefits of spironolactone [58]. However subsequent analysis showed that participants with an LVEF at the lower end of the spectrum showed greater potential benefit from spironolactone with respect to the primary endpoint (cardiovascular death, HF hospitalisation, resuscitated sudden death) and to heart failure hospitalisations [59]. A post-hoc analysis of the same study revealed significant and unanticipated differences in outcome rates and clinical characteristics between patients enrolled from Russia and Georgia and those from the Americas (United States, Canada, Brazil, and Argentina), such that interpretation of the former may be inconsistent. In the Americas group treatment with spironolactone was associ- ated with reduced risk of the primary composite outcome, cardiovascular mortality, and hospitalisation for heart failure [60].

Treatment with MRAs may be problematic for patients with CKD because of adverse events, particularly hyperkalaemia; a quarter of the Americas cohort enrolled in TOPCAT developed a potassium $\geq 5.5 \mathrm{mmol} / \mathrm{L}$. Those treated with spironolactone were also significantly more likely to have a doubling of creatinine during follow-up [60]. Similar consequences are seen in trials of spironolactone in patients with CKD and HFrEF [61]. Of note, TOPCAT excluded patients with eGFR $<30 \mathrm{~mL} / \mathrm{min} / 1.73 \mathrm{~m}^{2}$, for whom such adverse events would be more serious.

A later analysis of the 1175 TOPCAT participants with a recorded baseline urine albumin: creatinine ratio (UACR) showed that increased uACR was associated with worse clinical outcomes, with no difference between the spironolactone and placebo arms. However, the group treated with spironolactone had reduced albuminuria after one year, and reduction in albumin excretion was associated with a reduction in heart failure hospitalisation and all-cause mortality [62]. Naturally there was an association between increased albuminuria and CKD. Analysis of the same cohort by kidney function (eGFR 30-45, 45-60, $\geq 60 \mathrm{~mL} / \mathrm{min} / 1.73$ $\mathrm{m}^{2}$ ) showed that at all levels of CKD considered spironolactone did lower the risk of reaching the primary composite endpoint. However, spironolactone was also associated with increased adverse events including hyperkalaemia and worsening renal function, leading the authors to conclude that spironolactone should only be initiated in patients with advanced CKD if close monitoring is available [37]. 
Table 2. Summary of pharmacological treatment options in heart failure with preserved ejection fraction (HFpEF) and renal considerations.

\begin{tabular}{lll}
\hline Class of agent & Effect on HFpEF & Renal considerations \\
\hline Loop diuretics & Symptomatic benefit when treating fluid overload & Possible association with greater decline in renal function \\
\hline Renin-angiotensin-aldosterone & Prognostic benefit not demonstrated [54,55] & Can potentially slow CKD progression via blood pressure \\
system inhibitors & control and reduction of proteinuria; \\
& May precipitate renal hypoperfusion; \\
& Risk of hyperkalaemia \\
\hline
\end{tabular}

Mineralocorticoid receptor Mortality benefit not demonstrated [58]
antagonists (MRAs)

Reduction of albuminuria [62];

antagonists (MRAs)

Lower risk of composite endpoint of cardiovascular mortality, heart failure hospitalisation or aborted cardiac arrest in those with estimated GFR $30-60 \mathrm{~mL} / \mathrm{min} / 1.73 \mathrm{~m}^{2}$ [37];

May be associated with reduced hospitalisations Risk of adverse events including hyperkalaemia and for heart failure [58] worsening renal function [60]

No reduction in composite endpoint of death $\quad$ No differing treatment effect between those with eGFR

Sacubitril-valsartan from cardiovascular causes or hospitalisations for heart failure [35]

$30-60$ or $>60 \mathrm{~mL} / \mathrm{min} / 1.73 \mathrm{~m}^{2}$ [35];

Reduction in renal composite endpoint $(\geq 50 \%$ decrease in eGFR, development of end-stage renal disease, or death from renal causes) when in combination with MRA, compared to MRA + valsartan alone [65]

\begin{tabular}{lll}
\hline Phosphodiesterase-5 inhibitors No clinical benefit [7] & Associated with worsening renal function [7] \\
\hline $\begin{array}{l}\text { Sodium zirconium } \\
\text { cyclosilicate Patiromer }\end{array}$ & $\begin{array}{l}\text { No specific benefits } \\
\text { Heart failure patients included in trial [72,73] } \\
\text { May allow other medications to be tolerated }\end{array}$ & Effective treatment for hyperkalaemia [74,75] \\
\hline $\begin{array}{lll}\text { Sodium-glucose } \\
\text { cotransporter 2 inhibitors }\end{array}$ & $\begin{array}{l}\text { Reduction in cardiovascular mortality and } \\
\text { hospitalisations for heart failure [76] }\end{array}$ & $\begin{array}{l}\text { Promising results in CKD without heart failure [77]; } \\
\text { DELIVER trial ongoing }\end{array}$ \\
\hline
\end{tabular}
eGFR, estimated glomerular filtration rate; MRA, mineralocorticoid receptor antagonist; CKD, chronic kidney disease.

\subsection{Sacubitril (neprilysin inhibitor)-valsartan}

The primary outcome in the PARAGON-HF trial comparing sacubitril-valsartan with valsartan alone in 4796 patients with HFpEF was a composite of total hospitalisations for heart failure and death from cardiovascular causes, and rates were not significantly different between the two groups [35]. A pooled analysis with data from the PARADIGM-HF trial (inclusion LVEF $<40 \%$ ) suggested a beneficial effect for treatment with sacubitril-valsartan for participants with a "mid-range" LVEF, without definition of a specific threshold [63]. Entry criteria for PARAGON-HF included LVEF $\geq 45 \%$, but also a high NT-proBNP cut-off, to the effect that participants my not reflect the milder end of HFpEF disease [64].

The rate ratio in PARAGON-HF appeared to favour sacubitril-valsartan in participants with a baseline eGFR $<60 \mathrm{~mL} / \mathrm{min} / 1.73 \mathrm{~m}^{2}$ [relative risk (RR) $0.79,95 \% \mathrm{CI}$ $0.66-0.95$ ] but equivocal for those with baseline eGFR $\geq 60$ $\mathrm{mL} / \mathrm{min} / 1.73 \mathrm{~m}^{2}$ (1.01 (0.8-1.27), suggesting that those with CKD saw benefit from the intervention. However, after consideration in a multivariable model changes in eGFR were not found to be associated with differing treatment effect.
A sub-group analysis of PARAGON-HF looked at the findings according to background of MRA therapy. Jering et al. [65] found potential value in combining treatment with MRA and sacubitril/valsartan in patients with HFpEF. The proportion of patients reaching the secondary outcome renal composite endpoint ( $\geq 50 \%$ decrease in eGFR, development of ESRD, or death from renal causes) was comparable between groups with and without baseline MRA use, and lower with sacubitril/valsartan compared to valsartan alone. Patients with combined sacubitril/valsartan and baseline MRA use had lower eGFR decline, without compromised safety outcomes such as hypotension and hyperkalaemia, but only $24 \%$ of the PARAGON-HF cohort also took an MRA. The U.S. Food and Drug Administration (FDA) approved the single pill combination of sacubitril and valsartan, for treatment of HFpEF in early 2021. Of note, a small study of 21 patients with HFpEF and on regular PD has shown short-term symptomatic and biochemical benefits of sacubitril/valsartan in this cohort too [66].

\subsection{Phosphodiesterase-5 inhibitors}

Another treatment that has been considered is sildenafil, a phosphodiesterase-5 inhibitor. The 
Phosphodiesterase-5 Inhibition to Improve Clinical Status and Exercise Capacity in Heart Failure with Preserved Ejection Fraction (RELAX) trial compared sildenafil with placebo in $212 \mathrm{HFpEF}$ patients (with LVEF $\geq 50 \%$ ), of whom 114 had CKD. Over a limited follow-up period (24 weeks) sildenafil was associated with worsening renal function [7], and is not recommended.

\subsection{Ultrafiltration}

Ultrafiltration has been considered for management of advanced heart failure without ESRD. Grossekettler et al. [67] have shown symptomatic improvement and reduced hospitalisations in patients with undifferentiated (but mean EF 31\%) heart failure and non-end-stage CKD treated with peritoneal ultrafiltration. Subsequent sub-group analysis demonstrated that the beneficial effects were greater among those with HFpEF than HFrEF [68]. The CARESS$\mathrm{HF}$ trial investigated venous ultrafiltration as treatment for acute decompensated heart failure (any EF) with worsened renal failure and found no benefits but increased adverse events compared to standard diuretic treatment [69].

\subsection{Potassium management}

Maintaining safe potassium levels is a particular concern for patients with HFpEF and CKD, and use of various treatments under consideration has had to be tempered as a result. Interestingly, potassium abnormalities appear to pose a greater threat for patients with HFpEF than HFrEF [70]. Hyperkalaemia is common in advanced CKD, and extreme abnormalities of potassium in either direction can cause arrhythmias. There appears to be a U-shaped relationship between potassium and mortality, with normokalaemia offering the best prognosis. Ferriera et al. [43], conducting a post-hoc analysis of the PARAGON-HF trial, observed that in patients with HFpEF taking either valsartan or sacubitril/valsartan those with potassium outside the range 4-5 $\mathrm{mmol} / \mathrm{L}$ had higher rates of heart failure hospitalisations. Hypokalaemia carried a greater risk than hyperkalaemia, and was also associated with increased risk of death within the follow-up period for patients with CKD. Patients with potassium $>5.2 \mathrm{mmol} / \mathrm{L}$ were excluded from the trial, so hyperkalaemia effects are likely underrepresented. The authors concluded that potassium abnormalities likely reflect more advanced HFpEF and intensive treatment (especially with diuretics) rather than directly causing adverse outcomes.

The recent emergence of patiromer and sodium zirconium cyclosilicate (SZC), as effective treatment strategies for hyperkalaemia, should allow more patients to start or remain on medications such as RAAS inhibitors or MRAs. These are newer potassium binders with improved efficacy and safety profile, compared to their predecessor sodium polystyrene sulfonate. The two work at different sites in the gastrointestinal tract resulting in increased potassium faecal excretion and, hence, reduced serum potassium lev- els. Given their individual characteristics it has been proposed that patiromer might be preferable for the management of chronic hyperkalaemia with SZC a better option for the acute management of hyperkalaemia [71].

Both agents appear efficacious and reasonably well tolerated in patients with heart failure (with any EF) and though there is no available evidence in relation to $\mathrm{HFpEF}$ specifically, there is no reason that their effects would be compromised [72-74].

\subsection{Future treatments}

Sodium-glucose cotransporter 2 (SGLT2) inhibitors offer hope to improve prognosis for many patients with HFpEF and CKD, especially those with diabetes. There is robust evidence supporting a prognostic benefit from SGLT2 inhibition in HFrEF which is independent of the presence of diabetes [78-80], and as a result empagliflozin and dapagliflozin have recently been endorsed in the management of HFrEF by international organisations [81,82]. At the same time, SGLT2 inhibitors have been associated with improved renal outcomes in both high cardiovascular risk and CKD cohorts, an effect which, again, extends to non-diabetic populations [83-85]. Recently, the results of EMPEROR PRESERVED were published [76] making empagliflozin the first pharmacological agent ever reported to confer a prognostic benefit in HFpEF. This trial enrolled 5988 patients with NYHA class II-IV heart failure and an EF of more than $40 \%$, assigned to receive empagliflozin or placebo on top of standard therapy. Patients on empagliflozin had a lower risk of the primary outcome of cardiovascular death or hospitalisation for heart failure with a HR of 0.79 (95\% CI $0.69-0.90 ; p<0.001)$ compared to placebo, which, appeared principally driven by a reduction in the risk for hospitalisation for heart failure and was independent of the presence of diabetes. Analysis of prespecified subgroups showed that the lower risk of primary outcome held in the groups with LVEF $<50 \%$ or $\geq 50 \%$ to $<60 \%$, but not in the group with LVEF $\geq 60 \%$. While the eGFR decline was slower in the empagliflozin group $\left(-1.25\right.$ vs. $\left.-2.62 \mathrm{~mL} / \mathrm{min} / 1.73 \mathrm{~m}^{2} ; p<0.001\right)$, there was no difference in the composite renal outcome between the two groups. However, when re-analysed defining a renal event using conventional meta-analysis criteria (including $\geq 50 \%$ sustained decline in eGFR instead of $\geq 40 \%$ ) the hazard ratio for the effect of empagliflozin on renal events in the subgroup of those with ejection fraction $41-49 \%$ became significant: 0.41 (95\% confidence interval $0.20-0.85)$ [86]. The ongoing Dapagliflozin Evaluation to Improve the LIVEs of Patients with Preserved Ejection Fraction Heart Failure (DELIVER) randomised controlled trial is comparing dapagliflozin $10 \mathrm{mg}$ once per day with placebo in patients with $\mathrm{HF}$ and LVEF $>40 \%$, and includes patients with eGFR $\geq 25 \mathrm{~mL} / \mathrm{min} / 1.73 \mathrm{~m}^{2}$ [87]. Effect on slope of eGFR will be assessed as an exploratory endpoint, and 6263 participants have been recruited and randomised. 
Other potential pharmacological agents to be explored include growth hormone-releasing agonists, which appear to improve diastolic function in a swine model of CKDinduced HFpEF [24]. The current Optimize-HFpEF trial is exploring the impact of systematically screening for and treating co-morbidities seen commonly in HFpEF, working from the hypothesis that these co-morbidities, including renal, contribute to the widespread pro-inflammatory state that leads to development on HFpEF [88]. Unfortunately, specific treatments for CKD caused by the most common aetiologies (hypertension and diabetes) are lacking, although of course the underlying conditions may be optimally managed. There may also be potential for future development of treatment options that specifically target inflammation and the subsequent pathways to fibrosis [4], or the various pathways by which CKD leads to coronary microvascular dysfunction [89]. Further, as noted when considering specific treatments above, trials have been undertaken adopting a range of LVEF thresholds, and as such results must be interpreted with a degree of caution when extrapolating to specific patient populations. Stark dichotomisation of HFpEF and HFrEF may not necessarily be helpful, especially given HFpEF phenotype heterogeneity.

\section{Acute kidney injury}

Acute Kidney Injury (AKI) is defined by a sudden deterioration in renal function with rapid increase in serum creatinine or decrease in urine output [90], and is common in patients admitted to hospital for treatment of acute decompensation of heart failure (ADHF). A large registry study in the USA found that AKI complicated $20 \%$ of two million admissions with ADHF patients without underlying CKD [91], and slightly more commonly seen in HFrEF than in HFpEF.

In a pooled analysis of four studies of HFpEF patients admitted to hospital with ADHF, baseline renal dysfunction, defined as eGFR $<60 \mathrm{~mL} / \mathrm{min} / 1.73 \mathrm{~m}^{2}$ ) was present in $82 \%$ of participants [92]. Calculating from the figures presented, AKI occurred in $22 \%$ of admissions. Obese patients admitted with ADHF were younger, had higher blood pressure and were more likely to have diabetes, but had similar baseline kidney function to non-obese counterparts. During comparable diuresis obese patients were significantly more likely to suffer AKI.

It is difficult to be sure whether transient creatinine changes during diuresis reflect changing haemodynamics within the glomerulus or true organ damage. An analysis of undifferentiated (i.e., both HFrEF and HFpEF) patients undergoing aggressive diuresis during ADHF did not find associations between worsening renal function and recognised markers of renal tubular injury including neutrophil gelatinase-associated lipocalin (NGAL) and kidney injury molecule 1 (KIM-1) [93]. Worsening renal function during ADHF is associated with echocardiographic signs of increased right ventricular free wall thickness and right ven- tricular dysfunction [42].

AKI in general is associated with future development of CKD [94], although long-term prognosis following AKI in HFpEF is not yet demonstrated. While in an undifferentiated cohort admitted with ADHF worsening renal function was associated with significantly worse in hospital mortality, complication rate and length of stay [95], in a pure HFpEF cohort of 332 patients (including with and without CKD) 30 day outcomes were not affected by development of AKI [92]. Patients with HFpEF with normal baseline renal function admitted with ADHF who developed AKI had an increased in-hospital mortality: $4.9 \%$ vs. $1.6 \%$, adjusted odds ratio (OR) 3.21, $p \leq 0.001$ [91].

Cardiac catheterization, commonly used for diagnostic and therapeutic reasons, carries a risk of post-procedure AKI. While there are clear recommendations for preprocedure hydration to mitigate AKI risk in patients without heart failure, and at a lower rate for those with HFrEF, the little evidence available to date suggests that over-hydration is not helpful for those with HFpEF [96].

\section{Conclusions}

HFpEF and kidney dysfunction commonly co-exist, and cardiologists, nephrologists and community physicians will care for patients with both with increasing frequency. They commonly share multiple risk factors and specific pathophysiological features of each contribute to the development and progression of the other, to the extent that they may often be seen as different manifestations of the same disease spectrum. Patients with a dual diagnosis of HFpEF and CKD appear to bear a more severe clinical picture and a worse prognosis. Neither CKD as caused by diabetes/hypertension/obesity nor HFpEF currently have proven treatments beyond control of co-morbidities and management of symptoms, and prevention and limitation of progression are key. The emerging evidence of a potential promising role of SGLT2 inhibitors in the management of HFpEF, on the background of their already demonstrated renal prognostic benefit may significantly add to the limited existing strategies, and possibly provide valuable insights on disease pathophysiology, should targeted research be taken forward.

\section{Author contributions}

JJ wrote the draft. EL conceived the idea and edited. EA edited the final version of the manuscript. All authors read and approved the final version of the manuscript.

\section{Ethics approval and consent to participate}

Not applicable.

\section{Acknowledgment}

Not applicable. 


\section{Funding}

This study received no external funding.

\section{Conflict of interest}

The authors declare no conflict of interest. Eirini Lioudaki and Emmanuel Androulakis are serving as the Guest editor of this journal. We declare that Eirini Lioudaki had no involvement in the peer review of this article and has no access to information regarding its peer review. Full responsibility for the editorial process for this article was delegated to Giuseppe Coppolino.

\section{References}

[1] Dunlay SM, Roger VL, Redfield MM. Epidemiology of heart failure with preserved ejection fraction. Nature Reviews Cardiology. 2017; 14: 591-602.

[2] Pieske B, Tschöpe C, de Boer RA, Fraser AG, Anker SD, Donal $\mathrm{E}$, et al. How to diagnose heart failure with preserved ejection fraction: the HFA-PEFF diagnostic algorithm: a consensus recommendation from the Heart Failure Association (HFA) of the European Society of Cardiology (ESC). European Heart Journal. 2019; 40: 3297-3317.

[3] GBD Chronic Kidney Disease Collaboration. Global, regional, and national burden of chronic kidney disease, 1990-2017: a systematic analysis for the Global Burden of Disease Study 2017. Lancet. 2020; 395: 709-733.

[4] Paulus WJ, Zile MR. From Systemic Inflammation to Myocardial Fibrosis. Circulation Research. 2021; 128: 1451-1467.

[5] Dini FL, Demmer RT, Simioniuc A, Morrone D, Donati F, Guarini $\mathrm{G}$, et al. Right ventricular dysfunction is associated with chronic kidney disease and predicts survival in patients with chronic systolic heart failure. European Journal of Heart Failure. 2012; 14: 287-294.

[6] Unger ED, Dubin RF, Deo R, Daruwalla V, Friedman JL, Medina $\mathrm{C}$, et al. Association of chronic kidney disease with abnormal cardiac mechanics and adverse outcomes in patients with heart failure and preserved ejection fraction. European Journal of Heart Failure. 2016; 18: 103-112.

[7] Patel RB, Mehta R, Redfield MM, Borlaug BA, Hernandez AF, Shah SJ, et al. Renal Dysfunction in Heart Failure with Preserved Ejection Fraction: insights from the RELAX Trial. Journal of Cardiac Failure. 2020; 26: 233-242.

[8] Shah SJ, Katz DH, Selvaraj S, Burke MA, Yancy CW, Gheorghiade M, et al. Phenomapping for novel classification of heart failure with preserved ejection fraction. Circulation. 2015; 131: 269-279.

[9] Zelnick LR, Katz R, Young BA, Correa A, Kestenbaum BR, de Boer IH, et al. Echocardiographic Measures and Estimated GFR Decline among African Americans: the Jackson Heart Study. American Journal of Kidney Diseases. 2017; 70: 199-206.

[10] Ravera M, Noberasco G, Signori A, Re M, Filippi A, Cannavò $\mathrm{R}$, et al. Left-ventricular hypertrophy and renal outcome in hypertensive patients in primary-care. American Journal of Hypertension. 2013; 26: 700-707.

[11] Zile MR, Gottdiener JS, Hetzel SJ, McMurray JJ, Komajda M, McKelvie R, et al. Prevalence and significance of alterations in cardiac structure and function in patients with heart failure and a preserved ejection fraction. Circulation. 2011; 124: 2491-2501.

[12] Melenovsky V, Borlaug BA, Rosen B, Hay I, Ferruci L, Morell $\mathrm{CH}$, et al. Cardiovascular features of heart failure with preserved ejection fraction versus nonfailing hypertensive left ventricular hypertrophy in the urban Baltimore community: the role of atrial remodeling/dysfunction. Journal of the American College of Cardiology. 2007; 49: 198-207.

[13] Lam CSP, Roger VL, Rodeheffer RJ, Bursi F, Borlaug BA, Ommen SR, et al. Cardiac structure and ventricular-vascular function in persons with heart failure and preserved ejection fraction from Olmsted County, Minnesota. Circulation. 2007; 115: 1982-1990.

[14] Hsiao S, Chiou K. Renal function decline predicted by left atrial expansion index in non-diabetic cohort with preserved systolic heart function. European Heart Journal Cardiovascular Imaging. 2017; 18: 521-528.

[15] Gan GCH, Bhat A, Kadappu KK, Fernandez F, Gu KH, Chen HHL, et al. Usefulness of Left Atrial Strain to Predict End Stage Renal Failure in Patients with Chronic Kidney Disease. The American Journal of Cardiology. 2021; 151: 105-113.

[16] Guazzi M, Ghio S, Adir Y. Pulmonary Hypertension in HF$\mathrm{pEF}$ and HFrEF. Journal of the American College of Cardiology. 2020; 76: 1102-1111.

[17] Vanderpool RR, Saul M, Nouraie M, Gladwin MT, Simon MA. Association between Hemodynamic Markers of Pulmonary Hypertension and Outcomes in Heart Failure with Preserved Ejection Fraction. JAMA Cardiology. 2018; 3: 298-306.

[18] Issa N, Krowka MJ, Griffin MD, Hickson LJ, Stegall MD, Cosio FG. Pulmonary hypertension is associated with reduced patient survival after kidney transplantation. Transplantation. 2008; 86: 1384-1388.

[19] Zlotnick DM, Axelrod DA, Chobanian MC, Friedman S, Brown $\mathrm{J}$, Catherwood E, et al. Non-invasive detection of pulmonary hypertension prior to renal transplantation is a predictor of increased risk for early graft dysfunction. Nephrology, Dialysis, Transplantation. 2010; 25: 3090-3096.

[20] Agrawal A, Naranjo M, Kanjanahattakij N, Rangaswami J, Gupta S. Cardiorenal syndrome in heart failure with preserved ejection fraction - an under-recognized clinical entity. Heart Failure Reviews. 2019; 24: 421-437.

[21] Klein DA, Katz DH, Beussink-Nelson L, Sanchez CL, Strzelczyk TA, Shah SJ. Association of Chronic Kidney Disease with Chronotropic Incompetence in Heart Failure with Preserved Ejection Fraction. The American Journal of Cardiology. 2015; 116: $1093-1100$.

[22] Lo KB, Mezue K, Ram P, Goyal A, Shah M, Krishnamoorthy $\mathrm{P}$, et al. Echocardiographic and Hemodynamic Parameters Associated with Diminishing Renal Filtration among Patients with Heart Failure with Preserved Ejection Fraction. Cardiorenal Medicine. 2019; 9: 83-91.

[23] Brouwers FP, de Boer RA, van der Harst P, Voors AA, Gansevoort RT, Bakker SJ, et al. Incidence and epidemiology of new onset heart failure with preserved vs. reduced ejection fraction in a community-based cohort: 11-year follow-up of PREVEND. European Heart Journal. 2013; 34: 1424-1431.

[24] Rieger AC, Bagno LL, Salerno A, Florea V, Rodriguez J, Rosado $\mathrm{M}$, et al. Growth hormone-releasing hormone agonists ameliorate chronic kidney disease-induced heart failure with preserved ejection fraction. Proceedings of the National Academy of Sciences of the United States of America. 2021; 118: e2019835118.

[25] Hickson LJ, Negrotto SM, Onuigbo M, Scott CG, Rule AD, Norby SM, et al. Echocardiography Criteria for Structural Heart Disease in Patients with End-Stage Renal Disease Initiating Hemodialysis. Journal of the American College of Cardiology. 2016; 67: 1173-1182.

[26] Paulus WJ, Tschöpe C. A novel paradigm for heart failure with preserved ejection fraction: comorbidities drive myocardial dysfunction and remodeling through coronary microvascular endothelial inflammation. Journal of the American College of Cardiology. 2013; 62: 263-271.

[27] Mihai S, Codrici E, Popescu ID, Enciu A, Albulescu L, Necula 
LG, et al. Inflammation-Related Mechanisms in Chronic Kidney Disease Prediction, Progression, and Outcome. Journal of Immunology Research. 2018; 2018: 2180373.

[28] Upadhya B, Amjad A, Stacey RB. Optimizing the Management of Obese HFpEF Phenotype: can we Mind both the Heart and the Kidney? Journal of Cardiac Failure. 2020; 26: 108-111.

[29] Faul C, Amaral AP, Oskouei B, Hu M, Sloan A, Isakova T, et al. FGF23 induces left ventricular hypertrophy. The Journal of Clinical Investigation. 2011; 121: 4393-4408.

[30] Neumann J, Ligtenberg G, Klein II, Koomans HA, Blankestijn PJ. Sympathetic hyperactivity in chronic kidney disease: pathogenesis, clinical relevance, and treatment. Kidney International. 2004; 65: 1568-1576.

[31] Nickel NP, O’Leary JM, Brittain EL, Fessel JP, Zamanian RT, West JD, et al. Kidney dysfunction in patients with pulmonary arterial hypertension. Pulmonary Circulation. 2017; 7: 38-54.

[32] Chapter 1: Definition and classification of CKD. Kidney International Supplement . 2013; 3: 19-62.

[33] Lejeune S, Roy C, Slimani A, Pasquet A, Vancraeynest D, Beauloye $\mathrm{C}$, et al. Heart failure with preserved ejection fraction in Belgium: characteristics and outcome of a real-life cohort. Acta Cardiologica. 2020; 76: 697-706.

[34] Tromp J, Teng T, Tay WT, Hung CL, Narasimhan C, Shimizu $\mathrm{W}$, et al. Heart failure with preserved ejection fraction in Asia. European Journal of Heart Failure. 2019; 21: 23-36.

[35] Solomon SD, McMurray JJV, Anand IS, Ge J, Lam CSP, Maggioni AP, et al. Angiotensin-Neprilysin Inhibition in Heart Failure with Preserved Ejection Fraction. New England Journal of Medicine. 2019; 381: 1609-1620.

[36] Jackson CE, Solomon SD, Gerstein HC, Zetterstrand S, Olofsson B, Michelson EL, et al. Albuminuria in chronic heart failure: prevalence and prognostic importance. The Lancet. 2009; 374 : 543-550.

[37] Beldhuis IE, Myhre PL, Claggett B, Damman K, Fang JC, Lewis $\mathrm{EF}$, et al. Efficacy and Safety of Spironolactone in Patients with HFpEF and Chronic Kidney Disease. JACC: Heart Failure. 2019; 7: 25-32.

[38] Antlanger M, Aschauer S, Kopecky C, Hecking M, Kovarik JJ, Werzowa J, et al. Heart Failure with Preserved and Reduced Ejection Fraction in Hemodialysis Patients: Prevalence, Disease Prediction and Prognosis. Kidney \&Amp; Blood Pressure Research. 2017; 42: 165-176.

[39] Chisavu LA, Apostol A, Pop GN, Ivan V, Schiller O, Bob F, et $a l$. Exploring the relation between mortality and left ventricular structure and function in stable hemodialysis treated patients, a longitudinal multicenter cohort study. Scientific Reports. 2021; 11: 12694.

[40] Zhong Y, Cai Y, Liu M, Bai W, Wang F, Tang H, et al. Left ventricular diastolic pressure gradient and outcome in advanced chronic kidney disease patients with preserved ejection fraction. The International Journal of Cardiovascular Imaging. 2021; 37: 2663-2673.

[41] Wang AY, Wang M, Lam CW, Chan IH, Lui S, Sanderson JE. Heart failure with preserved or reduced ejection fraction in patients treated with peritoneal dialysis. American Journal of Kidney Diseases. 2013; 61: 975-983.

[42] Mukherjee M, Sharma K, Madrazo JA, Tedford RJ, Russell SD, Hays AG. Right-Sided Cardiac Dysfunction in Heart Failure with Preserved Ejection Fraction and Worsening Renal Function. The American Journal of Cardiology. 2017; 120: 274-278.

[43] Ferreira JP, Claggett BL, Liu J, Desai AS, Pfeffer MA, Anand IS, et al. Serum potassium and outcomes in heart failure with preserved ejection fraction: a post-hoc analysis of the PARAGONHF trial. European Journal of Heart Failure. 2021; 23: 776-784.
[44] Segar MW, Patel RB, Patel KV, Fudim M, DeVore AD, Martens $\mathrm{P}$, et al. Association of Visit-to-Visit Variability in Kidney Function and Serum Electrolyte Indexes With Risk of Adverse Clinical Outcomes Among Patients With Heart Failure With Preserved Ejection Fraction. Journal of the American Medical Association Cardiology. 2021; 6: 68-77.

[45] Chirinos JA, Orlenko A, Zhao L, Basso MD, Cvijic ME, Li Z, et al. Multiple Plasma Biomarkers for Risk Stratification in Patients with Heart Failure and Preserved Ejection Fraction. Journal of the American College of Cardiology. 2020; 75: 12811295.

[46] Katz DH, Burns JA, Aguilar FG, Beussink L, Shah SJ. Albuminuria is independently associated with cardiac remodeling, abnormal right and left ventricular function, and worse outcomes in heart failure with preserved ejection fraction. JACC: Heart Failure. 2014; 2: 586-596.

[47] Oeun B, Hikoso S, Nakatani D, Mizuno H, Suna S, Kitamura T, et al. Prognostic significance of dipstick proteinuria in heart failure with preserved ejection fraction: insight from the PURSUITHFpEF registry. BMJ Open. 2021; 11: e049371.

[48] Miura M, Shiba N, Nochioka K, Takada T, Takahashi J, Kohno $\mathrm{H}$, et al. Urinary albumin excretion in heart failure with preserved ejection fraction: an interim analysis of the CHART 2 study. European Journal of Heart Failure. 2012; 14: 367-376.

[49] Chen S, Huang J, Su H, Chiu Y, Chang J, Hwang S, et al. Prognostic Cardiovascular Markers in Chronic Kidney Disease. Kidney \&Amp; Blood Pressure Research. 2018; 43: 1388-1407.

[50] Gergei I, Krämer BK, Scharnagl H, Stojakovic T, März W. Renal function, N-terminal Pro-B-Type natriuretic peptide, propeptide big-endothelin and patients with heart failure and preserved ejection fraction. Peptides. 2019; 111: 112-117.

[51] Reddy YNV, Obokata M, Dean PG, Melenovsky V, Nath KA, Borlaug BA. Long-term cardiovascular changes following creation of arteriovenous fistula in patients with end stage renal disease. European Heart Journal. 2017; 38: 1913-1923.

[52] Khan YH, Sarriff A, Adnan AS, Khan AH, Mallhi TH. Chronic Kidney Disease, Fluid Overload and Diuretics: A Complicated Triangle. PLoS One. 2016; 11: e0159335.

[53] Schwartzenberg S, Redfield MM, From AM, Sorajja P, Nishimura RA, Borlaug BA. Effects of Vasodilation in Heart Failure with Preserved or Reduced Ejection Fraction. Journal of the American College of Cardiology. 2012; 59: 442-451.

[54] Massie BM, Carson PE, McMurray JJ, Komajda M, McKelvie R, Zile MR, et al. Irbesartan in patients with heart failure and preserved ejection fraction. The New England Journal of Medicine. 2008; 359: 2456-2467.

[55] Damman K, Perez AC, Anand IS, Komajda M, McKelvie RS, Zile MR, et al. Worsening renal function and outcome in heart failure patients with preserved ejection fraction and the impact of angiotensin receptor blocker treatment. Journal of the American College of Cardiology. 2014; 64: 1106-1113.

[56] Yusuf S, Pfeffer MA, Swedberg K, Granger CB, Held P, McMurray JJV, et al. Effects of candesartan in patients with chronic heart failure and preserved left-ventricular ejection fraction: the CHARM-Preserved Trial. Lancet. 2003; 362: 777-781.

[57] Lund LH, Claggett B, Liu J, Lam CS, Jhund PS, Rosano GM, et al. Heart failure with mid-range ejection fraction in CHARM: characteristics, outcomes and effect of candesartan across the entire ejection fraction spectrum. European Journal of Heart Failure. 2018; 20: 1230-1239.

[58] Pitt B, Pfeffer MA, Assmann SF, Boineau R, Anand IS, Claggett $\mathrm{B}$, et al. Spironolactone for heart failure with preserved ejection fraction. The New England Journal of Medicine. 2014; 370: 1383-1392. 
[59] Solomon SD, Claggett B, Lewis EF, Desai A, Anand I, Sweitzer $\mathrm{NK}$, et al. Influence of ejection fraction on outcomes and efficacy of spironolactone in patients with heart failure with preserved ejection fraction. European Heart Journal. 2016; 37: 455-462.

[60] Pfeffer MA, Claggett B, Assmann SF, Boineau R, Anand IS, Clausell N, et al. Regional variation in patients and outcomes in the Treatment of Preserved Cardiac Function Heart Failure with an Aldosterone Antagonist (TOPCAT) trial. Circulation. 2015; 131: 34-42.

[61] Khan MS, Khan MS, Moustafa A, Anderson AS, Mehta R, Khan SS. Efficacy and Safety of Mineralocorticoid Receptor Antagonists in Patients with Heart Failure and Chronic Kidney Disease. The American Journal of Cardiology. 2020; 125: 643-650.

[62] Selvaraj S, Claggett B, Shah SJ, Anand I, Rouleau JL, O'Meara $\mathrm{E}$, et al. Prognostic Value of Albuminuria and Influence of Spironolactone in Heart Failure with Preserved Ejection Fraction. Circulation: Heart Failure. 2018; 11: e005288.

[63] Solomon SD, Vaduganathan M, L. Claggett B, Packer M, Zile $\mathrm{M}$, Swedberg K, et al. SacubitrilValsartan across the Spectrum of Ejection Fraction in Heart Failure. Circulation. 2020; 141: 352-361.

[64] Gronda E, Vanoli E, Iacoviello M. The PARAGON-HF trial: the sacubitril/valsartan in heart failure with preserved ejection fraction. European Heart Journal Supplements. 2020; 22: L77-L81.

[65] Jering KS, Zannad F, Claggett B, Mc Causland FR, Ferreira JP, Desai A, et al. Cardiovascular and Renal Outcomes of Mineralocorticoid Receptor Antagonist Use in PARAGON-HF. JACC: Heart Failure. 2021; 9: 13-24.

[66] Fu S, Xu Z, Lin B, Chen J, Huang Q, Xu Y, et al. Effects of Sacubitril-Valsartan in Heart Failure With Preserved Ejection Fraction in Patients Undergoing Peritoneal Dialysis. Frontiers in Medicine. 2021; 8: 657067.

[67] Grossekettler L, Schmack B, Meyer K, Brockmann C, Wanninger R, Kreusser MM, et al. Peritoneal dialysis as therapeutic option in heart failure patients. European Society of Cardiology Heart Failure. 2019; 6: 271-279.

[68] Grossekettler L, Schmack B, Brockmann C, Wanninger R, Kreusser MM, Frankenstein L, et al. Benefits of peritoneal ultrafiltration in $\mathrm{HFpEF}$ and HFrEF patients. BMC Nephrology. 2020; 21: 179.

[69] Bart BA, Goldsmith SR, Lee KL, Givertz MM, O'Connor CM, Bull DA, et al. Ultrafiltration in decompensated heart failure with cardiorenal syndrome. The New England Journal of Medicine. 2012; 367: 2296-2304.

[70] Savarese G, Xu H, Trevisan M, Dahlström U, Rossignol P, Pitt $\mathrm{B}$, et al. Incidence, Predictors, and Outcome Associations of Dyskalemia in Heart Failure with Preserved, Mid-Range, and Reduced Ejection Fraction. JACC: Heart Failure. 2019; 7: 6576.

[71] Shrestha DB, Budhathoki P, Sedhai YR, Baniya R, Cable CA, Kashiouris MG, et al. Patiromer and Sodium Zirconium Cyclosilicate in Treatment of Hyperkalemia: a Systematic Review and Meta-Analysis. Current Therapeutic Research. 2021; 95: 100635.

[72] Anker SD, Kosiborod M, Zannad F, Piña IL, McCullough PA, Filippatos G, et al. Maintenance of serum potassium with sodium zirconium cyclosilicate (ZS-9) in heart failure patients: results from a phase 3 randomized, double-blind, placebocontrolled trial. European Journal of Heart Failure. 2015; 17: 1050-1056.

[73] Piña IL, Yuan J, Ackourey G, Ventura H. Effect of patiromer on serum potassium in hyperkalemic patients with heart failure: Pooled analysis of 3 randomized trials. Progress in Cardiovascular Diseases. 2020; 63: 656-661.

[74] Roger S, Spinowitz B, Lerma E, Singh B, Packham D, Al-
Shurbaji A, et al. Efficacy and Safety of Sodium Zirconium Cyclosilicate for Treatment of Hyperkalemia: an 11-Month Open-Label Extension of HARMONIZE. American Journal of Nephrology. 2019; 50: 473-480.

[75] Kosiborod M, Peacock WF, Packham DK. Sodium zirconium cyclosilicate for urgent therapy of severe hyperkalemia. The New England Journal of Medicine. 2015; 372: 1577-1578.

[76] Anker SD, Butler J, Filippatos G, Ferreira JP, Bocchi E, Bohm $\mathrm{M}$, et al. Empagliflozin in Heart Failure with a Preserved Ejection Fraction. The New England Journal of Medicine. 2021; 385: 1451-1461.

[77] Heerspink HJL, Stefánsson BV, Correa-Rotter R, Chertow GM, Greene T, Hou F, et al. Dapagliflozin in Patients with Chronic Kidney Disease. The New England Journal of Medicine. 2020; 383: 1436-1446.

[78] Zannad F, Ferreira JP, Pocock SJ, Anker SD, Butler J, Filippatos $\mathrm{G}$, et al. SGLT2 inhibitors in patients with heart failure with reduced ejection fraction: a meta-analysis of the EMPERORReduced and DAPA-HF trials. The Lancet. 2020; 396: 819-829.

[79] Anker SD, Butler J, Filippatos G, Khan MS, Marx N, Lam CSP, et al. Effect of Empagliflozin on Cardiovascular and Renal Outcomes in Patients with Heart Failure by Baseline Diabetes Status: Results From the EMPEROR-Reduced Trial. Circulation. 2021; 143: 337-349.

[80] McMurray JJV, Solomon SD, Inzucchi SE, Kober L, Kosiborod MN, Martinez FA, et al. Dapagliflozin in Patients with Heart Failure and Reduced Ejection Fraction. The New England Journal of Medicine. 2019; 381: 1995-2008.

[81] McDonagh TA, Metra M, Adamo M, Gardner RS, Baumbach A, Bohm M, et al. 2021 ESC Guidelines for the diagnosis and treatment of acute and chronic heart failure. European Heart Journal. 2021; 42: 3599-3726.

[82] Maddox TM, Januzzi JL, Allen LA, Breathett K, Butler J, Davis LL, et al. 2021 Update to the 2017 ACC Expert Consensus Decision Pathway for Optimization of Heart Failure Treatment: Answers to 10 Pivotal Issues about Heart Failure with Reduced Ejection Fraction. Journal of the American College of Cardiology. 2021; 77: 772-810.

[83] McMurray JJV, Wheeler DC, Stefánsson BV, Jongs N, Postmus $\mathrm{D}$, Correa-Rotter R, et al. Effect of Dapagliflozin on Clinical ssOutcomes in Patients with Chronic Kidney Disease, with and without Cardiovascular Disease. Circulation. 2021; 143: 438448.

[84] Neal B, Perkovic V, Matthews DR. Canagliflozin and Cardiovascular and Renal Events in Type 2 Diabetes. The New England Journal of Medicine. 2017; 377: 2099.

[85] Wanner C, Inzucchi SE, Zinman B. Empagliflozin and Progression of Kidney Disease in Type 2 Diabetes. The New England Journal of Medicine. 2016; 375: 1801-1802.

[86] Packer M, Zannad F, Butler J, Filippatos G, Ferreira JP, Pocock SJ, et al. Influence of endpoint definitions on the effect of empagliflozin on major renal outcomes in the EMPERORPreserved trial. European Journal of Heart Failure. 2021; 23: 1798-1799.

[87] Solomon SD, de Boer RA, DeMets D, Hernandez AF, Inzucchi $\mathrm{SE}$, Kosiborod MN, et al. Dapagliflozin in heart failure with preserved and mildly reduced ejection fraction: rationale and design of the DELIVER trial. European Journal of Heart Failure. 2021; 23: 1217-1225.

[88] Fu M, Zhou J, Thunström E, Almgren T, Grote L, Bollano E, et al. Optimizing the Management of Heart Failure with Preserved Ejection Fraction in the Elderly by Targeting Comorbidities (OPTIMIZE-HFPEF) Journal of Cardiac Failure. 2016; 22: 539-544.

[89] van de Wouw J, Broekhuizen M, Sorop O, Joles JA, Verhaar MC, Duncker DJ, et al. Chronic Kidney Disease as a Risk Factor 
for Heart Failure with Preserved Ejection Fraction: a Focus on Microcirculatory Factors and Therapeutic Targets. Frontiers in Physiology. 2019; 10: 1108.

[90] Khwaja A. KDIGO clinical practice guidelines for acute kidney injury. Nephron. Clinical Practice. 2012; 120: c179-c184.

[91] Doshi R, Dhawan T, Rendon C, Rodriguez MA, Al-khafaji JF, Taha M, et al. Incidence and implications of acute kidney injury in patients hospitalized with acute decompensated heart failure. Internal and Emergency Medicine. 2020; 15: 421-428.

[92] Reddy YNV, Obokata M, Testani JM, Felker GM, Tang WHW, Abou-Ezzeddine OF, et al. Adverse Renal Response to Decongestion in the Obese Phenotype of Heart Failure with Preserved Ejection Fraction. Journal of Cardiac Failure. 2020; 26: 101107.

[93] Ahmad T, Jackson K, Rao VS, Tang WHW, Brisco-Bacik MA, Chen $\mathrm{HH}$, et al. Worsening Renal Function in Patients with Acute Heart Failure Undergoing Aggressive Diuresis is not As- sociated with Tubular Injury. Circulation. 2018; 137: $2016-$ 2028.

[94] Coca SG, Singanamala S, Parikh CR. Chronic kidney disease after acute kidney injury: a systematic review and meta-analysis. Kidney International. 2012; 81: 442-448.

[95] Forman DE, Butler J, Wang Y, Abraham WT, O'Connor CM, Gottlieb SS, et al. Incidence, predictors at admission, and impact of worsening renal function among patients hospitalized with heart failure. Journal of the American College of Cardiology. 2004; 43: 61-67.

[96] Bei WJ, Wang K, Li HL, Lin KY, Guo XS, Chen SQ, et al. Safe Hydration Volume to Prevent Contrast-induced Acute Kidney Injury and Worsening Heart Failure in Patients With Heart Failure and Preserved Ejection Fraction After Cardiac Catheterization. Journal of Cardiovascular Pharmacology. 2017; 70: 168175. 